\title{
Notes on Groundwater Age in Forward and Inverse Modeling
}

\author{
Timothy R. Ginn • Hanieh Haeri • Arash Massoudieh • \\ Laura Foglia
}

Received: 26 March 2008 / Accepted: 15 April 2009 / Published online: 3 July 2009

(C) The Author(s) 2009. This article is published with open access at Springerlink.com

\begin{abstract}
Several example applications of the groundwater age equation are used to derive some basic results about age in aquifers and to draw linkages among published results involving temporal moments and aquifer-aquitard diffusive mass transfer. We then describe a brief numerical study of an inverse problem in which hydraulic conductivities are identified using both piezometric head and groundwater age data where inclusion of age data helps to reduce evidential nonuniqueness. This reflects the global representation of flow process contained in age data, and that the value of age data in inverse identification of flow properties depends on the paths taken by the sampled groundwater.
\end{abstract}

Keywords Groundwater age · Dispersion · Transport · Inverse problems

\section{Introduction}

Understanding groundwater age involves isotope geochemistry and fractionation, interfacial reactive geochemistry, recharge and surface-subsurface hydrology on long time scales, and both groundwater flow and advective-dispersive-diffusive transport in heterogeneous materials. The characterization of groundwater age is of practical value because some aspects of the distribution of groundwater age are measurable through analyses of dissolved species such as anthropogenic and cosmogenic radioisotopes and industrial contaminants such as chloroflouorocarbons. Clarke and Fritz (1997) provide a detailed background on the methods for chemical analyses of anthropogenic, cosmogenic, or geogenic tracers appearing in groundwater that can serve as indicators of age of groundwater. These include principally

Paper presented at PORO2007, the 7th North American Workshop on Applications of the Physics of Porous Media, Puerto Vallarta, Mexico, 2-6 November, 2007.

T. R. Ginn $(\bowtie) \cdot$ H. Haeri · A. Massoudieh · L. Foglia

Department of Civil \& Environmental Engineering, University of California at Davis,

Davis, CA 95616, USA

e-mail: trginn@ucdavis.edu

URL: http://cee.engr.ucdavis.edu/faculty/ginn/ 
radioactive tracers whose measurement along with knowledge of their activities in recharge waters historically (often linked to atmospheric concentrations) give a radiometric age. Varni and Carrera (1998) summarize issues involved in interpreting age from tracers and make the case for solute-independent modeling of groundwater ages.

Groundwater age modeling provides a means to link such data to subsurface hydraulic and geochemical properties and processes. Studies focusing on particular aspects of groundwater age (e.g., recharge estimation and fractionation) appear in various literatures with different mathematical/conceptual foundations. Variously, age is inferred from concentrations of single isotopes/tracers that are supposed to reflect the mean age; age is inferred from multiple isotope/tracer concentrations that together represent different components of age ("young water" and "old water"); age is supposed to arise from lumped-parameter (assumed age distribution) models; age is supposed to arise from two-dimensional compartment models; age is supposed to arise from detailed three-dimensional flow models. These variations result from ad hoc specifications of governing equations that are site- and case-specific, that are not all rooted in the same fundamental equation, and that are not often evaluated in a relative comparison of multiple possible models of a given situation. Also the linkage between measured concentrations of isotopes (one or many) and age moments varies, as reviewed in Varni and Carrera (1998). Such inconsistency would be unacceptable in the more self-consistent contexts of groundwater flow or solute contaminant transport in which fundamental governing equations are established if debated. In the context of groundwater age however this inconsistency is a feature of the interface between perspectives of groundwater age: geochemical based and hydrological based. One objective of this article is to help resolve the inconsistency in fundamental conceptual-mathematical modeling of groundwater age in the hydrological-based perspective. We review the equation for groundwater age distributions and analyze this equation under several simplified conditions. We define "age mass" in terms of this distribution and we identify a few important results about groundwater age in simplified flows, including the fact that it is always at least inverse-Gaussian distributed and that steady-state age moments are already available for many cases involving mobile-immobile diffusive mass transfer.

Another objective of this study is to explore the importance of groundwater age data to the model calibration and how it can help with the reduction in parameter estimation nonuniqueness. In order to apply effectively distributed parameter flow and transport models in groundwater systems, the model parameters, presuming they exist, must be known, and due to inaccessibility parameter values be specified via solution of the inverse problem of groundwater flow and solute transport. One avenue to reduction of nonuniqueness involves use of multiple data sets governed by the same aquifer property to be estimated. Since head distribution is a result of diffusion governed by hydraulic conductivity and boundary conditions, and age distribution is a result of advection-dispersion governed by hydraulic conductivity and porosity and boundary conditions, their joint use in inversion may sharpen inverse problem solutions. The coupling of piezometric head and concentration data has been used for many years to estimate groundwater hydraulic parameters (Sun and Yeh 1990; Cheng and Yeh 1992; Sun 1994; Anderman et al. 1996; Weiss and Smith 1998; Wen et al. 2002; Hill and Tiedeman 2007). Since groundwater age is affected by the same processes as those controlling solute transport, it can be considered as a tracer. Age tracers such as environmental and radioactive isotopes (e.g. tritium/helium, ${ }^{36} \mathrm{Cl} / \mathrm{Cl},{ }^{14} \mathrm{C} / \mathrm{C}$ ) and their history of decay in subsurface can serve as proxy for some measure of groundwater age. Such observation data have been used in the same way to help in estimation of aquifer properties (Reilly et al. 1994; Sheets et al. 1998; Portniaguine and Solomon 1998; Zhu 2000; Weissmann et al. 2002; Reynolds and Marimuthu 2007). In the inverse study presented here, we apply modeling of both flow and transport in an idealized 2-D problem in the forward sense to solve simultaneously the 
steady-state piezometric head and mean age distributions, the filtered output of which is used as input to an indirect inversion scheme to calibrate some of the parameters involved. The objective is to understand how the addition of observation data density and type (e.g., head, flow rate, and age) can improve the inverse solution and potentially reduce its inherent nonuniqueness.

\section{Background and Governing Equation for Groundwater Age}

Here, we provide a brief review of conceptual and mathematical models designed for the quantification of the spatiotemporal distribution of the groundwater age distribution and/or its moments. We restrict ourselves to general models developed from basic balance equations and do not include site-specific models developed in ad hoc fashion. Early conceptual models of groundwater age arise in part from residence time considerations in chemical engineering (e.g., Danckwerts 1953; Spaulding 1958; Randolph 1964) and appear in hydrogeology in Maloszewski and Zuber (1982) with focus on lumped parameter models (e.g., assumed age distributions with fittable parameters, Maloszewski and Zuber 2002; Cook and Böhlke 2000; Ivey et al. 2008). Subsequent technical works on groundwater age are numerous and beyond the present scope but for a couple highlights. The need for improved models for simulation of measurable groundwater age as opposed to purely kinematic age (mixing ignored) is discussed by Campaña (1987) who developed one of the early models that treats the entire distribution of groundwater age and not just the mean age; the groundwater flow is treated via compartment model. Goode (1996) and Varni and Carrera (1998) established the equation for mean and moments of groundwater age, respectively. Later Weissmann et al. (2002) modeled the distribution of ground water ages indicated by chlorofluorocarbons in a heterogeneous aquifer using particle tracing and echoed the need for the complete groundwater age distribution. In aquifer characterization by inverse problem solution, some early uses of groundwater age in analogy to tracer concentration data appears in Reilly et al. (1994), Sheets et al. (1998), and Portniaguine and Solomon (1998), and such coupled inverses are now common (e.g., Reynolds and Marimuthu 2007).

\subsection{The Groundwater Age Equation}

The governing equation for groundwater age is (Ginn 1999)

$$
\frac{\partial n \rho}{\partial t}+\nabla \cdot(\mathbf{v} n \rho)-\nabla \cdot[\mathbf{D} n \nabla \rho]+1 \frac{\partial n \rho}{\partial a}=0
$$

where $\rho=\rho(\mathbf{x}, a, t)$ is the conventional space- and time-dependent mass density of water now also distributed over age $a, \mathbf{v}$ is the conventional Darcy flux divided by the porosity, $\mathbf{D}$ is the conventional dispersion-diffusion tensor, $n$ is the porosity, and we assume no internal sources or sinks (recharge and withdrawals may be included but here are treated via boundary condition for simplicity). The velocity may vary in space and time (Ginn 1999, equation 10, contrary to the claim of Cornaton and Perrochet 2007). This mass balance expression governs the distribution of age by advecting water mass density along the age dimension at unit velocity. Mixing in age arises from nonuniform velocity $\mathbf{v}$ and from dispersive and diffusive mixing controlled by $\mathbf{D}$. Thus, age distribution is governed by an advection-dispersion equation in four dimensions (space and age), where transport in the age dimension is with unit velocity and zero dispersion-diffusion. There is no diffusion in the $a$ direction because there is no difference between the aging rate of individual water molecules as there is in the transport rate 
of individual water molecules (Ginn 1999, appendix). Boundary conditions are set by considering that water entering the domain requires now a specified age (zero for surface recharge if one ignores residence time in the vadoze zone). In that case the specified flux boundary condition as position $\mathbf{x}^{\prime}$ is placed at $\mathbf{x}=\mathbf{x}^{\prime}, a=0$. For instance, if water enters an aquifer at an upstream recharge boundary, the age equation above is complemented with a Dirichlet boundary condition that specifies water density at that boundary as a Dirac distribution in $a^{+}$.

\subsection{On a Continuum Mechanical Basis for Groundwater Age}

Here we make a few observations on the conceptual basis for modeling groundwater age, when viewed from the continuum-mechanical basis. A sample of groundwater includes a set of water molecules which (ignoring hydrolysis effects by their mere fraction) each have a clearly defined "age" since entering the subsurface. Thus a bulk (ground)water-phase sample is intrinsically distributed over age, and within any continuum-mechanical approach there is no choice but to treat groundwater age as a deterministic distribution. This perspective induces self-consistent representations (e.g., Goode 1996; Varni and Carrera 1998; Ginn 1999) that recognize the distributed nature of groundwater age in the mathematical treatment. When one writes the actual governing equation for this distribution one is forced to identify the initial and boundary conditions for the mass balance of groundwater over space, time and age. Indeed in application we cannot easily prove that an aquifer's ages are in steady-state and so the initial condition may matter to current ages. That is, simulating groundwater age distributions requires specification of initial conditions that may involve connate waters or other imaginations reaching back to the creation of the actual subsurface which is far beyond our conceptual model's limitations. However this challenge is one that arises from characterization limitations and not from the fundamental model that is unambiguous.

Also we note that the age-mass is so far only loosely defined without rigorous definition of how the underlying distribution of water density over age enters the definition. Age-mass is defined as the product of "mean age times water mass" (Goode 1996), or "the product of mass and age" (e.g., Bethke and Johnson 2002), or as water density times its age (e.g., Cornaton and Perrochet 2006). In fact volumetric age mass is the un-normalized first moment of the age distribution (un-normalized because the distribution need not integrate to unity)

$$
\bar{a}(\mathbf{x}, t)=\int_{0}^{\infty} a \rho(\mathbf{x}, t, a) \mathrm{d} a
$$

and its relation to mean age $A$ is $A=\bar{a} / \rho^{\mathrm{T}}$ where $\rho^{\mathrm{T}}=\int_{0}^{\infty} \rho(\mathbf{x}, t, a) \mathrm{d} a$ is the conventional water density (Ginn 1999). For incompressible flows $\rho^{\mathrm{T}}=1$ (SI units) and the volumetric age mass is equivalent to the mean age.

Finally we emphasize that the distribution of water mass over age is a deterministic, and not probabilistic one (Ginn 2007; Cornaton and Perrochet 2007). Uncertainty in the flow properties and their associated parameters have not yet been formally introduced into the analysis of the groundwater age problem, this is still an open research question.

\section{Several Illustrative Cases}

\subsection{Construction of Previous Age Equations from Equation (1)}

Previous differential equations for the transport of different measures of age include: the mean age equation of Goode (1996), the percentile age equation of Varni and Carrera (1998), 
as well as their arbitrary moment equation, and the idealized aquitard age equation used in Bethke and Johnson (2002). Each are derived from (1) as follows.

One obtains the mean age equation of Goode (1996) by multiplying (1) by $a$ (the age dimension) and integrating from zero to infinity, and defining age mass as above, $\bar{a}=$ $A \rho^{\mathrm{T}}=\int_{0}^{\infty} a \rho(a) \mathrm{d} a$ where $A$ is the conventional mean age, $\rho^{\mathrm{T}}$ is the conventional groundwater density, and $\rho(a)$ is the distribution of local groundwater density over age. It should be noted that in Ginn (1999) the porosity in the dispersion term is erroneously inside the gradient operator, the correct form of the mean age equation is

$$
\frac{\partial n \rho^{\mathrm{T}} A}{\partial t}+\nabla \cdot\left(\mathbf{v} n \rho^{\mathrm{T}} A\right)-\nabla \cdot\left[\mathbf{D} n \cdot \nabla \rho^{\mathrm{T}} A\right]-n \rho^{\mathrm{T}}=0
$$

Second, any definite integral of age (i.e., over the interval $\left[a_{1}, a_{2}\right]$ ) of Eq. 1 gives the governing equation for the fraction of water that is between $a_{1}$ and $a_{2}$ age as follows. Integration of (1) on $\left[a_{1}, a_{2}\right]$ gives

$$
\frac{\partial n P}{\partial t}+\nabla \cdot(\mathbf{v} n P)-\nabla \cdot[\mathbf{D} n \cdot \nabla P]+n \rho\left(a_{2}\right)-n \rho\left(a_{1}\right)=0
$$

where $P \equiv \int_{a_{1}}^{a_{2}} \rho(a) \mathrm{d} a$. In the steady-state case with $a_{1}=0$ and thus $\rho\left(a_{1}\right)=0$, recognizing that $n \rho\left(a_{2}\right)=\frac{\partial n P}{\partial a}\left(a_{2}\right)$, and defining $F=P / \rho^{\mathrm{T}}$ one obtains

$$
\frac{\partial n \rho^{\mathrm{T}} F}{\partial a_{1}}+\nabla \cdot\left(\mathbf{v} n \rho^{\mathrm{T}} F\right)-\nabla \cdot\left[\mathbf{D} n \cdot \nabla \rho^{\mathrm{T}} F\right]=0
$$

that is comparable to Eq. (2a) of Varni and Carrera (1998) for the percentile of age without source or sink term (by construction here) and with age replacing their time. These forms are not exactly equivalent. The Varni and Carrera model is for transient age, but has no age dimension, so age is defined as $t-\tau$ where $\tau$ is recharge time of the water and thus their equation represents a continuous family of equations for varying $\tau$. Additionally they specify two such families depending on the initial age of water. Equation 4 is singleton and as a steady state expression it does not depend on initial ages by construction. Equation 3, also singleton but with the added dimension of age, is the most general form and represents transient conditions in a single equation.

Third, the equation for any $m$ th moment of the age (Varni and Carrera 1998) distribution is obtained in analogy to the development of the mean age equation noted above but with multiplication by $a^{m}$ (age to the $m$ th power) instead of by $a$. Integration, again with integration by parts on the aging term, yields

$$
\frac{\partial n \rho^{\mathrm{T}} A_{m}}{\partial t}+\nabla \cdot\left(\mathbf{v} n \rho^{\mathrm{T}} A_{m}\right)-\nabla \cdot\left[\mathbf{D} n \cdot \nabla \rho^{\mathrm{T}} A_{m}\right]-n m \rho^{\mathrm{T}} A_{m-1}=0
$$

where $A_{m} \rho^{\mathrm{T}}=\bar{a}_{m}=\int_{0}^{\infty} a^{m} \rho(a) \mathrm{d} a$, and $A_{m}$ is the $m$ th moment of the age distribution.

Fourth, the mean age equations that form the basis of the "paradox of groundwater age" (Bethke and Johnson 2002, "BJ") arise from Eq. 1 and traversing the derivation forces explicit identification of each assumption required. Consider ideal aquifer-aquitard layers that are flat, homogenous, isotropic and of constant thickness. Figure 1 shows half aquifer and half aquitard domains of one layer pair. Assume that dispersion longitudinally in the aquifer is zero and that lateral dispersion is fast so that the water in the aquifer is well-mixed laterally.

Only diffusive transport occurs in the aquitard that is adjacent to the aquifer. The "paradox" is the result that the efflux of "age-mass" (here, the product of water density and mean age) from the aquitard is, at steady-state, independent of the mixing and transport rates of 
Fig. 1 Schematic of flow system for the aquifer-aquitard problem

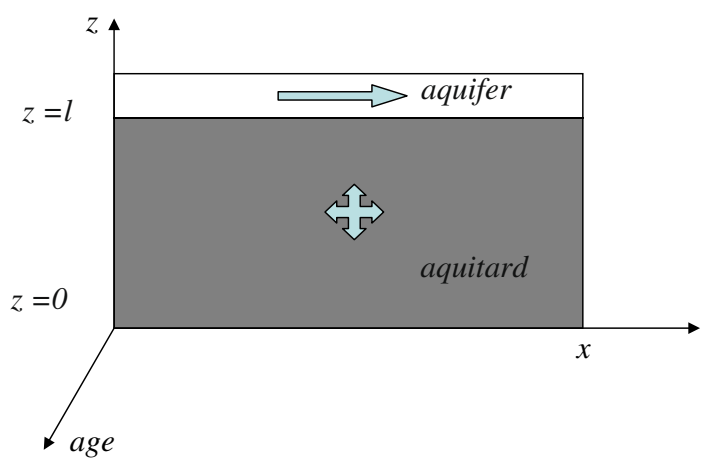

the water itself, and depends only on porosity and size of the aquitard. As will be shown an exact analogy of this result is previously obtained in temporal moments analysis of solute transport by Harvey and Gorelick (1995). This result is obtained by analysis of a governing equation written for the aquifer-aquitard system (although it can be obtained by simply forcing the age mass that accumulates in the aquitard to leave the aquitard, E. LaBolle, written communication), but without a clear foundation for the equations, that makes it hard to see the assumptions underlying the analyses.

To derive the BJ posited model let us call the aquifer domain " 1 " and the aquitard domain "2" and write Eq. 1 for each of the adjacent domains (Ginn 2000):

$$
\begin{gathered}
\frac{\partial n_{1} \rho_{1}}{\partial t}+1 \frac{\partial n_{1} \rho_{1}}{\partial a}+q \frac{\partial \rho_{1}}{\partial x}=- \text { loss }+ \text { gain } \\
\frac{\partial n_{2} \rho_{2}}{\partial t}+1 \frac{\partial n_{2} \rho_{2}}{\partial a}-D_{x} \frac{\partial^{2} n_{2} \rho_{2}}{\partial x^{2}}-D_{z} \frac{\partial^{2} n_{2} \rho_{2}}{\partial z^{2}}=\text { loss }- \text { gain }
\end{gathered}
$$

where $\rho_{1}$ and $\rho_{2}$ are distributions of water density over age in the aquifer and aquitard, respectively, and other terms as defined. The boundary conditions are: at $z=0, \frac{\partial \rho_{2}}{\partial z}=0$; at $z=l-$, we don't know; at $z=l+, x=0, \rho_{1}=\operatorname{Dirac}(a)$ representing entry of water in the upstream end of the aquifer. Assuming that the loss term is proportional to density $\rho_{1}$ and the gain term is proportional to density $\rho_{2}$ (a mass-action assumption), that the porosities are constants, that diffusion/dispersion in the x-direction is negligible in the aquitard, and then taking the first moment as above, we obtain

$$
\begin{aligned}
\frac{\partial a_{1}}{\partial t}-1+v \frac{\partial a_{1}}{\partial x} & =-\frac{k a_{1}}{n_{1}}+\frac{k a_{2}}{n_{1}} \\
\frac{\partial a_{2}}{\partial t}-1-D_{z} \frac{\partial^{2} a_{2}}{\partial z^{2}} & =\frac{k a_{1}}{n_{2}}-\frac{k a_{2}}{n_{2}}
\end{aligned}
$$

at $z=0, \frac{\partial a_{2}}{\partial z}=0$; at $z=l-$ we don't know; $z=l+, x=0, a_{1}=0$.

If we now assume steady state conditions, and also assume $k \rightarrow \infty$ so that equilibrium is always achieved between $a_{1}$ and $a_{2}(x, l-)$, the right-hand side of (7b) is zero and we have for the aquitard

$$
D_{z} \frac{\mathrm{d}^{2} a_{2}}{\mathrm{~d} z^{2}}+1=0
$$


at $z=0, \frac{\partial a_{2}}{\partial z}=0$; at $z=l-a_{2}(z=l-)=a_{1}$; at $z=l+, x=0, a_{1}=0$. Defining $\tau^{\prime}=a_{2}-a_{1}$,

$$
D_{z} \frac{\mathrm{d}^{2} \tau^{\prime}}{\mathrm{d} z^{2}}+1=0
$$

at $z=0, \frac{\partial \tau^{\prime}}{\partial z}=0$; at $z=l-, \tau^{\prime}(z=l-)=0$; at $z=l+, x=0, a_{1}=0$, the BJ system.

The system requires steady-state conditions (noted by BJ) but additionally the assumptions of constant porosity, complete lateral mixing in the aquifer, no diffusion in the aquitard parallel to the aquifer, and equilibrium between the aquifer and aquitard waters of any given age. The solution reveals that the aquitard's water age at the $z=0$ coordinate is the mean travel-time by diffusion from the aquifer to the aquitard midpoint, at steady-state. More generally, one may solve the more general equations to examine other aspects of the system such as time-to-steady-state. This is important for understanding how perturbations in aquifer flow (such as induced by climate change, pumping withdrawals, or artificial groundwater recharge) affect groundwater age distributions the moments of which may be measured in pumping or withdrawal wells. Such equations are tractable and can be solved as shown in Woolfenden and Ginn (2009) and in the next section.

\section{Steady-State Age in a One-dimensional Transport Field}

Here we show that age in any given sample of groundwater taken from the subsurface is in the simplest case of homogeneous steady flow at least inverse-Gaussian distributed, and further that the steady state first moments of age are available for a range of mobile-immobile domain configurations. Under steady state $1 \mathrm{D}$ constant porosity, velocity and dispersivity conditions, (1) becomes

$$
\frac{\partial \rho}{\partial a}+v \frac{\partial \rho}{\partial x}-D \frac{\partial^{2} \rho}{\partial x^{2}}=0
$$

with the Dirac- $\delta$ boundary condition, $\rho(x=0, a)=\delta^{+}(a)$. This is recognized as the classical $1 \mathrm{D}$ advection-dispersion equation with $a$ playing the role of $t$, with instantaneous pulse (of water) applied at age zero. The well-known solution is the inverse-Gaussian distribution (e.g., Simmons 1982; Hathhorn 1996),

$$
\rho(x, a)=\frac{x}{\sqrt{4 \pi D a^{3}}} \exp \left(\frac{-(v a-x)^{2}}{4 D a}\right)
$$

By comparison the solution under the "plug flow" assumption is the Dirac- $\delta$ spike at age satisfying $v a=x$. Thus the distribution of age is always at least inverse-Gaussian distributed, and simplifications below this description such as the plug-flow assumption are neither appropriate nor needed. This distribution is skewed with variance proportional to dispersion coefficient that can be large when sampled water reflects velocities that exhibit large variance ("macrodispersion"). If the mixing occurs at the sampling well and represents contributions from individual paths $i$ with fluxes $q_{i}$, then the groundwater age distribution of the sample is the flux-weighting of the individual inverse-Gaussian distributions (Ginn 2001). Finally it is important to note that the age distribution is calculable under multidimensional transient conditions with nonuniform flow and mixing, via (1), and so it is no longer necessary to ignore.

More generally it is straightforward to incorporate aging in multi-domain transport models. As shown here and expanded upon below, augmenting the original equations with the 
aging term allows not only calculation of age distributions reflecting any multidomain diffusion, it also provides a formal link to existing temporal moment expressions that are shown to be age moment expressions. Here we highlight the classical two-domain case. The classical two-domain model in $1 \mathrm{D}$, augmented with aging is

$$
\begin{gathered}
\frac{\partial n_{\mathrm{m}} \rho_{\mathrm{m}}}{\partial t}+\frac{\partial v n_{\mathrm{m}} \rho_{\mathrm{m}}}{\partial x}-\frac{\partial}{\partial x} n_{\mathrm{m}} D \frac{\partial \rho_{\mathrm{m}}}{\partial x}+1 \frac{\partial n_{\mathrm{m}} \rho_{\mathrm{m}}}{\partial a}=-\frac{n_{\mathrm{m}}}{n_{\mathrm{im}}} k\left(\rho_{\mathrm{m}}-\rho_{\mathrm{im}}\right) \\
\frac{\partial n_{\mathrm{im}} \rho_{\mathrm{im}}}{\partial t}+1 \frac{\partial n_{\mathrm{im}} \rho_{\mathrm{m}}}{\partial a}=k\left(\rho_{\mathrm{m}}-\rho_{\mathrm{im}}\right)
\end{gathered}
$$

where subscript $\mathrm{m}$ indicates mobile zone, subscript im indicates immobile zone, $n$ is porosity, $k$ is an exchange rate, other terms are as previously defined, and the velocities of aging in both equations are unity for demonstration but either can be "turned off" to determine the distribution over age in the mobile (or immobile) zone alone. At steady state again the age derivatives play the role of time derivatives and with the same Dirac- $\delta$ instantaneous (in age) pulse, the solution for the distribution of water over age in the mobile domain is given by the well-known Lassey (1988) solution corrected (Lassey 1989), with age replacing the time variable:

$$
C\left(x^{\prime}, a^{\prime}\right)=\int_{0}^{a^{\prime}} \Gamma_{o} \Omega_{f}\left(x^{\prime}, y^{\prime}\right) \mathrm{e}^{-\kappa_{1} y^{\prime}-\kappa_{2}\left(a^{\prime}-y^{\prime}\right)}\left[\delta\left(a^{\prime}-y^{\prime}\right)+\kappa_{1} \kappa_{2} y^{\prime} I_{1}^{\prime}\left(\kappa_{1} \kappa_{2} y^{\prime}\left(a^{\prime}-y^{\prime}\right)\right)\right] \mathrm{d} y^{\prime}
$$

where $C\left(0^{+}, a^{\prime}\right)=\Gamma_{o} \delta\left(a^{\prime}\right)$ specifies the Dirac-d instantaneous pulse (of water), $x^{\prime}=x v / D$, $a^{\prime}=a v^{2} / D, \kappa_{1}=k D / v^{2} n_{\mathrm{m}}, \kappa_{2}=k D / v^{2} n_{\mathrm{im}}$, and $I_{1}^{\prime}(z) \equiv I_{1}(2 \sqrt{z}) / \sqrt{z}$, where $I$ is the modified Bessel function of the first kind. The function $\Omega_{f}\left(x^{\prime}, y^{\prime}\right)=\frac{x^{\prime}}{\sqrt{4 \pi y^{\prime 3}}} \exp \left(\frac{-\left(x^{\prime}-y^{\prime}\right)^{2}}{4 y^{\prime}}\right)$ is the dispersion kernel for flux-averaged density undergoing advection and Fickian dispersion and is recognized as the inverse-Gaussian distribution introduced above but here for dimensionless space and age. Then the Lassey solution is seen to be a convolution of delays due to reversible multidomain diffusion with the conventional diffusive-dispersive transport, incurring additional variance to the age distribution beyond that occurring in the inverse-Gaussian case. Without interdomain mass transfer, i.e., $k=0$, (13) reduces to (11) as follows:

$$
\begin{aligned}
C\left(x^{\prime}, a^{\prime}\right) & =\int_{0}^{a^{\prime}} \Gamma_{o} \Omega_{f}\left(x^{\prime}, y^{\prime}\right)\left[\delta\left(a^{\prime}-y^{\prime}\right)\right] \mathrm{d} y^{\prime} \\
& =\Gamma_{o} \Omega_{f}\left(x^{\prime}, a^{\prime}\right)=\Gamma_{o} \frac{x^{\prime}}{\sqrt{4 \pi a^{3}}} \exp \left(\frac{-\left(x^{\prime}-a^{\prime}\right)^{2}}{4 a^{\prime}}\right)
\end{aligned}
$$

Replacing the dimensioned variables, and replacing $\Gamma_{o}$ with $C_{o} v^{2} / D$ (factors arising from the change to dimensioned variables within the Dirac- $\delta$ function) yields Eq. 11 .

\section{Solute Transport Equation Characteristic Solutions are Steady State Groundwater Age Distributions; and Temporal Moments are Age Moments}

The last two examples show how we can treat characteristic solutions for breakthrough curves for solute transport as exact analogues for age distributions at steady state, as a result of the fact that the two cases give identical mathematical expressions. This is in fact generally 
true and is easily shown as follows. Restate (1) with a diffusion term, and at in steady state condition and one obtains

$$
\frac{\partial n \rho}{\partial a}+\nabla \cdot(\mathbf{v} n \rho)-\nabla \cdot[\mathbf{D} n \nabla \rho]=\text { Diffusive_exchange }
$$

that with the natural boundary condition of the Dirac- $\delta$ density of water at zero age at inflow locations, is equivalent to the solute transport equation with arbitrary diffusion term with instantaneous pulse in time, if we allow $a$ to play the role of $t$. As a consequence the temporal moments of the associated breakthrough curve solutions to solute transport are equivalent to the moments of the groundwater age, and this is sometimes noted (e.g., Harvey and Gorelick 1995; Varni and Carrera 1998). Equation 14 shows the mathematical basis.

Consequently the moments of the steady state age distribution, when they exist, are given by the temporal moments of the flux-averaged solute concentrations under instantaneous pulse injection, for arbitrary interdomain mass transfer including powerlaw. Varni and Carrera (1998) note that the (age) moment equations they obtain (i.e., Eq. 5 above) are the transient forms of the steady state (temporal) moment equations of Harvey and Gorelick (1995); however, Varni and Carrera (1998) leave out multidomain diffusion for simplicity. [The effects of diffusion are later reincorporated in an example showing that as long as the total porosity is constant, the (steady-state) mean ages are independent of the rate of diffusion, confirming the statement by Harvey and Gorelick (1995) that for temporal moments, "the first moment of arrival time depends on the distribution coefficient (of porosities for multidomain diffusion) but not on the actual rate coefficients..." Since the first moment of arrival time is the mean age in steady-state conditions, this statement means that the mean age depends on distribution of porosities but not rate coefficients for multidomain diffusion. This is the same statement cast later as a novel paradox by Bethke and Johnson (2002).]

Recognizing that steady state age moments, when they exist, are given by temporal flux moments allows us to use the temporal moment results from the solute transport literature as indicators of age moments. Congruently the governing equation for moments of (steady state) groundwater age are equivalent to the governing equations for temporal moments of characteristic solutions to solute transport in the same formations. For example Eq. 21 of Harvey and Gorelick (1995) that is the equation governing the first temporal moment of solute transport with linear diffusive transfer, with $\beta=0, m_{1}=A, m_{0}=1$, initial concentration in immobile zone $S_{o}=0$, constant porosity and incompressible flow, becomes the mean groundwater age equation of Goode (1996) at steady state (Eq. 2 above without time derivative).

As a result, age moment equations with arbitrary diffusive mass transfer at steady state are already available as the temporal flux moment equations for solute transport under analogous boundary conditions. This includes solutions for transient moments with multidimensional nonuniform flow. Continuing the example above, equation (21) of Harvey and Gorelick (1995) with nonzero $\beta$ is the governing moment equation for groundwater age at steady state, when linear diffusion occurs. More general diffusive mass transfer moment equations (e.g., Harvey and Gorelick 1995; Luo et al. 2008) translate similarly, typically requiring for the solute transport flux moment equation that initial concentration in immobile zone be zero, porosity be constant, and flow be incompressible.

\section{Numerical Analysis of Uniqueness in an Inverse Problem with Age}

We now turn to a view of the inverse problem in a related situation constructed from a classical two-dimensional flow field with lower conductivity at depth so that diffusive transport 


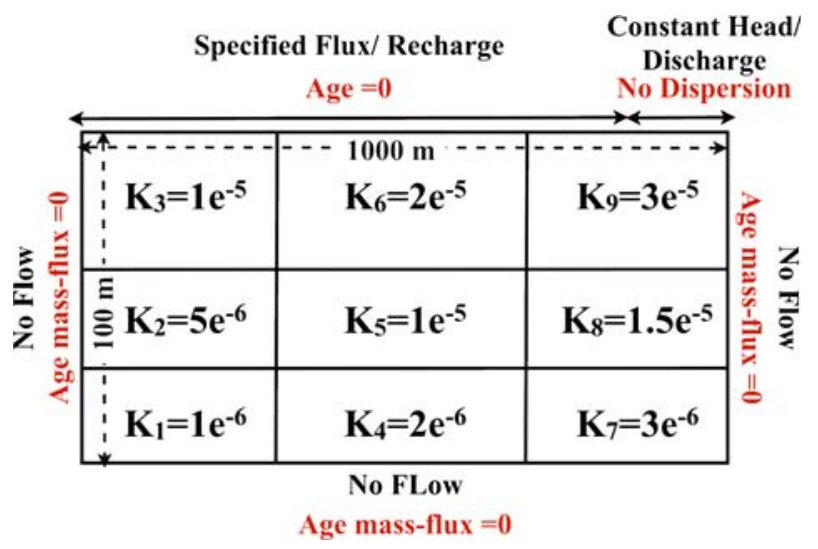

Fig. 2 Flow and age equation boundary conditions and the k-field for the studied aquifer system

plays a larger role with depth. The objective of this section is to examine how the inclusion of data reflecting mean groundwater age under simplified idealized conditions helps reduce (or not) nonuniqueness in a given inverse problem.

While it is possible to obtain core samples of subsurface materials and to perform laboratory experiments to determine parameter values on such samples, these values reflect flow and transport on the core-scale (centimeters). The model used for the real aquifer however almost always involves much larger units. The parameter values associated with the model grid scale are generally quite different from those defined on the core-scale samples. Hence, the value of additional characterization data used for groundwater model parameter identification depends strongly on its measurement scale (Scheibe and Chien 2003).

The identification of spatially-distributed hydrogeologic properties from hydraulic information or other data provide an alternative avenue toward characterization but one that is famously challenging due to nonuniqueness. One avenue to reduction of nonuniqueness involves use of multiple data sets, such as piezometric head, concentration and/or groundwater age, in the solution of the inverse problem. Since head information is governed by a diffusion equation and age information governed by an advection-dispersion-reaction equation, their coupling may sharpen inverse problem solutions.

We apply joint (flow and mean age) modeling for the forward solution of a simplified, idealized problem to solve simultaneously the steady-state flow and mean age equations, the output of which is used as input to an indirect inversion scheme, UCODE, to calibrate some of the parameters involved. The flow domain is a classical hypothetical two-dimensional system with three of four boundaries corresponding to no-flow conditions and the top boundary is modeled as a combination of constant head and recharge; this configuration is similar to those analyzed by Goode (1996) and earlier authors (Freeze and Witherspoon 1967). Figure 2 is a simple representation of the studied physical system, the boundary conditions for each of the equations and the k-field. Sensitivity analysis (including composite scaled sensitivity, CSS, dimensionless scaled sensitivity, DSS, and parameter correlation coefficient, pcc) and calibration have been carried out using UCODE_2005 (Poeter et al. 2005).

The physical properties of the aquifer are as follows: the hydraulic conductivities are decreasing with depth and coarsely heterogeneous as shown in Fig. 2, the porosity is 0.20 , the tortousity $(\tau)$ is 1 , the longitudinal dispersivity $\left(\alpha_{\mathrm{L}}\right)$ is $6.0 \mathrm{~m}$ and the transverse dispersivity $\left(\alpha_{\mathrm{T}}\right)$ is $0.6 \mathrm{~m}$, and the coefficient of molecular diffusion $\left(D_{\mathrm{m}}\right)$ equal to $1.16 \mathrm{e}-8 \mathrm{~m}^{2} / \mathrm{s}$. 
A full 2-D mechanical dispersion tensor has been used in this study built on the Darcy velocity (q) with the following diagonal components:

$$
D_{i i}=\alpha_{\mathrm{L}} \frac{q_{i}^{2}}{|\mathbf{q}|}+\alpha_{\mathrm{T}} \frac{q_{j}^{2}}{|\mathbf{q}|}+\tau n D_{\mathrm{m}}
$$

and off-diagonal components:

$$
D_{i j}=D_{j i}=\left(\alpha_{\mathrm{L}}-\alpha_{\mathrm{T}}\right) \frac{q_{i} q_{j}}{|\mathbf{q}|}+\tau n D_{\mathrm{m}}
$$

\subsection{Forward Solution of Flow and Mean Age Equations}

The spatially distributed hydraulic heads are determined for a steady state flow field and then used to derive the fluid velocity by differentiating the head field obtained. The flow equation solved is:

$$
\begin{array}{ll}
\nabla \cdot(K(x, y) h(x, y))=0 & \\
\mathbf{q}(x, y)=\mathbf{q}_{\mathrm{sp}}(x, y) & \text { on flux boundaries } \\
h(x, y)=h_{\mathrm{sp}}(x, y) & \text { on head boundaries }
\end{array}
$$

where $h(x, y)$ is the hydraulic head, $K(x, y)$ is the isotropic but heterogeneous hydraulic conductivity and $\mathbf{q}(x, y)$ is Darcy velocity.

Groundwater age is simulated using the mean age equation (Goode 1996):

$$
\begin{array}{ll}
1-\nabla \cdot[\mathbf{v}(x, y) A(x, y)]+\nabla \cdot[\mathbf{D}(x, y) \cdot \nabla A(x, y)]=0 & \\
A(x, y)=0 & \text { on inflow boundaries } \\
\mathbf{v}(x, y) A(x, y)-\mathbf{D}(x, y) \nabla A(x, y)=0 & \text { on outflow boundaries } \\
\mathbf{D}(x, y) \nabla A(x, y)=0 & \text { on no-flow boundaries }
\end{array}
$$

where $A(x, y)$ is the mean groundwater age, $\mathbf{D}$ is the dispersion tensor. The average pore velocity $(\mathbf{v}(x, y))$, is the ratio of Darcy velocity to porosity $(\theta)$. The mean age equation is a reactive transport equation that has a reaction rate of 1 that ages water by one unit of age per time.

The groundwater flow equation and the equation for mean groundwater age for the domain were both solved using a finite element solver COMSOL (2006). A direct linear solver UMFPACK was used as the forward solver. A Quadratic Lagrange Element shape function of order 2 was used for the basis functions. The test function is of the same type as the basis function so that the Galerkin method has been applied. The mesh elements are rectilinear with uniform intervals in the $x$ and $y$ direction, devised with a convergence analysis of grid resolution to address accuracy. The streamlines of the flow solution are shown in Fig. 3.

The results of the forward simulation showed that the maximum age occurs along the right boundary, with the greatest age at the bottom and decreasing toward the discharge boundary at the top (Fig. 4). This means that the age does not increase monotonically toward the discharge point as it might be expected. In fact the age increase monotonically toward the discharge boundary along each individual streamlines, but the "cell-averaged" ages do not increase in the same manner when the convergence of streamlines of very different lengths bring young and old water molecules to a single cell. The same result was shown in Goode (1996). As it was discussed by Goode (1996), this happens because "converging streamlines near the discharge boundary bring younger water into the cells along the right boundary. That is, the maximum age at a point is at the top right corner of the domain, but the average 


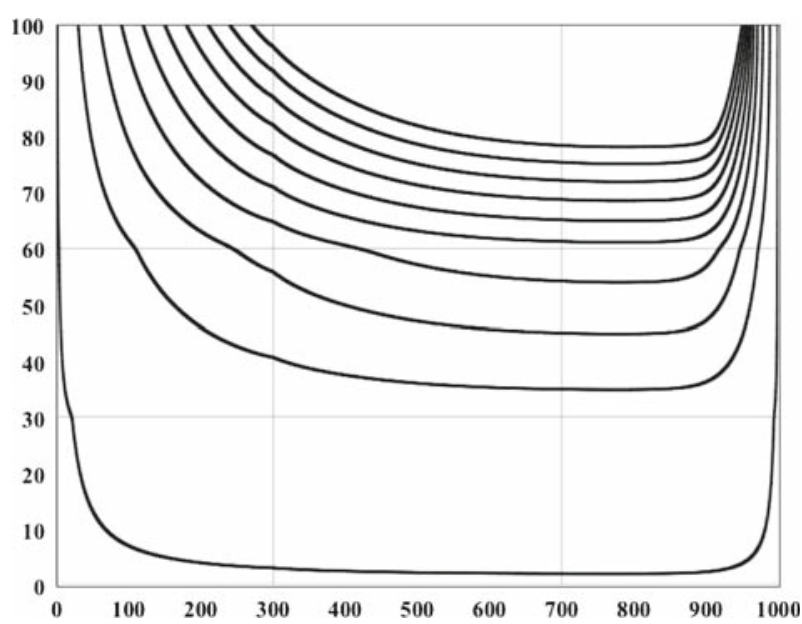

Fig. 3 Streamlines of flow in the studied aquifer system

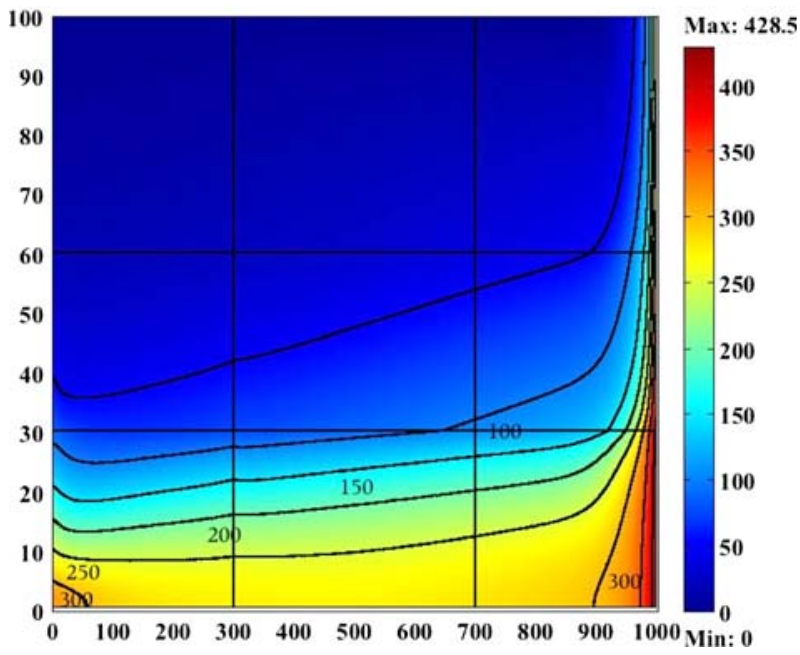

Fig. 4 Surface and contour plot of mean age distribution in the studied aquifer

age for the cell containing this point is less because of the convergence of streamlines in the cell."

\subsection{Inverse Solution of Coupled Piezometric and Age Equation}

\subsubsection{Parameter Estimation-UCODE}

UCODE-2005 was employed to calibrate key parameters of the physical system. UCODE-2005 estimates model parameters using a modified Gauss-Newton nonlinear regression technique. The modified Gauss-Newton technique minimizes a weighted least-squares 
objective function (Eq. 13) with respect to the parameter values. The objective function measures the fit of the simulated values to the observations:

$$
S(\mathbf{b})=\sum_{i=1}^{\mathrm{NH}} \omega_{h i}\left[y_{h i}-y_{h i}^{\prime}(\mathbf{b})\right]^{2}+\sum_{j=1}^{\mathrm{NA}} \omega_{a j}\left[y_{a j}-y_{a j}^{\prime}(\mathbf{b})\right]^{2}
$$

where $\mathbf{b}$ is a vector containing values of each of the parameters being estimated $(\mathrm{NP}=\mathrm{NH}+$ $\mathrm{NA}+\mathrm{NPR}$ ), NH is the number of head observations, NA is the number of age observations, NPR is the number of prior information values specified by the user, $y_{h i}$ and $y_{a j}$ are the $i$ th observed head and $j$ th observed age respectively being matched by the regression, $y_{h i}^{\prime}(\mathbf{b})$ is the simulated head that correspond to the $i$ th observed head (a function of $\mathbf{b}$ ), $y_{a j}^{\prime}(\mathbf{b})$ is the simulated age that correspond to the $j$ th observed age, and $\omega_{h i}$ and $\omega_{a j}$ are the weight for $i$ th head observation and $j$ th age observation respectively. In this study we specify the weights in terms of a variance with a value of 0.5 for head data and in terms of a coefficient of variation with a value of 0.1 for age observations. The errors are assumed independent and normally distributed. This weighting technique results in higher weights for younger water. Since the value of age data are generally higher than the head data in this study the weighting values presented above result in error residuals that are usually much higher for the age data than the head data, thus the error in simulated age become of high importance.

\subsubsection{Approach}

The goal of solving inverse problem in this study is to explore the reduction in nonuniqueness that results both from additional data from more observation locations and from combined use of piezometric head and groundwater mean age as opposed to exclusively piezometric head data. The observation points were chosen to be close to the ground surface (i.e., the top boundary) in order to represent the greater availability of near-surface data in the field (Fig. 5). The study involves parameter estimation with different number of observations of either only head or both head and mean age data. As the last part of the study some sampling points were added at depth in order to show the effect of data at regions intermediate to the flow stream (Fig. 5). Inverse modeling is then applied to these "data" in order to estimate the hydraulic conductivities under different observation types (head and mean age), densities (20, 10, and 6 observation points), patterns (concentrated or distributed) and accuracies (true or noisy). This results in 12 sampling scenarios:

- three with only head data at the top layer,

- three with both head and mean age data at the top layer,

- three with only head data at the top layer augmented by some observations at the bottom layer,

- three with both head and mean age data at the top layer augmented by some observations at the bottom layer

The study was performed under two observation accuracies for each observation scenario:

- 1st experiment: "true" observation values

Since the studied groundwater system is a synthetic case, the "true" head and age distribution (outcome of the forward solution given the true hydraulic conductivity field) was sub-sampled point wise (as discussed above) to obtain the "observation values" to be used for the solution of inverse problem. 


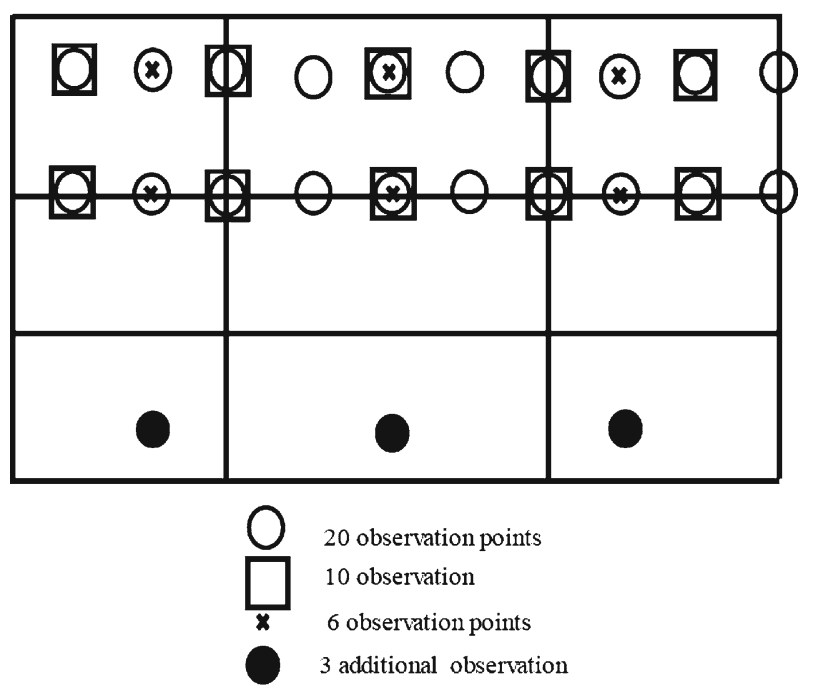

Fig. 5 Location of sampling sets for the three resolutions: "x" represents the 6 original sample locations; squares represents the next level involving 10 samples; circles represent the maximum resolution set involving 20 samples. All three sets involve either age and head, or just head data. Location distribution is skewed to the right in order to allow sampling to intercept high-density streamline regions to allow examination of effluent mean age. Note that this is not generally possible in realworld conditions. Finally the filled circles at the bottom indicate locations of deep samples examined for their utility in reducing nonuniqueness, and it was found (data not shown) that mean age data from these depths significantly reduced remaining nonuniqueness

- 2nd experiment: "noisy" observation values

In this experiment random noise (unbiased normally distributed noise) was imposed to the "true" pointwise head and age values of the 1st experiment and the same parameter estimation was performed to investigate how noise in the observations can affect the outcome of the inverse solution.

The goodness of fit and the error in estimated k-field compared to the true k-field were calculated in each sampling scenarios of each experiment to evaluate the nonuniqueness level in each observation set. To this aim, we calculated the mean squared error (MSE) - chosen here as one measure of nonuniqueness-between the original true zoned hydraulic conductivities shown in Fig. 2 and those determined by the inversion for each observation set (Eq. 14 ), with the constraint that the inverse-solved values correspond to insignificant error in the head and age fields as associated with each of the studied experiments $(<0.01 \%$, this value has been used to screen the solutions determined by inversion for best results).

$$
\mathrm{MSE}=\frac{\left.\sum_{i}^{n}\left\{K_{i} \text { (true }\right)-K_{i}(\text { estimated })\right\}^{2}}{n}, \quad n=\# \text { of conductivity zones }=9
$$

\subsection{Results and Discussion}

The final result is presented in Fig. 6 as a histogram of $\log (\mathrm{MSE})$ versus different experiments. A lower value of MSE for an observation set means that this measure of nonuniqueness is lower for that set compared to the others. The MSE values in this study are positive values smaller than 1, thus their natural logarithm values are negative. Smaller the MSE is, larger the $\log (\mathrm{MSE})$ absolute value is. Since Fig. 6 plots the $\log (\mathrm{MSE})$ for each observation set, higher 


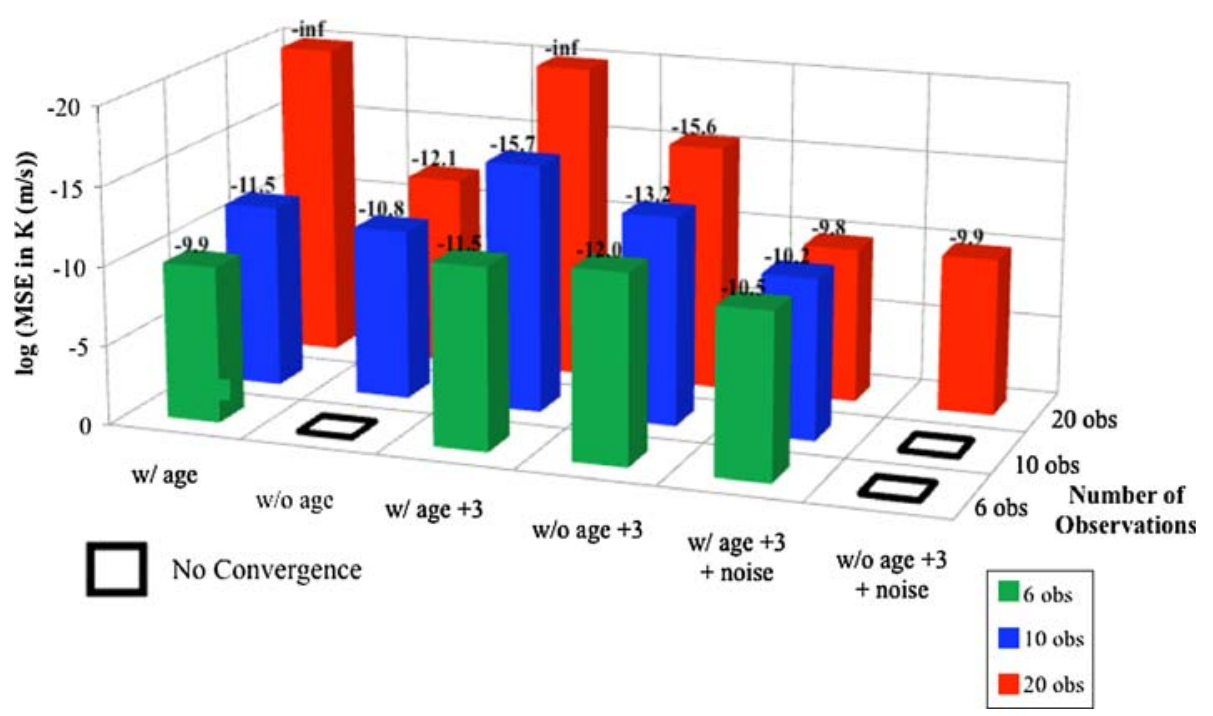

Fig. 6 Histogram showing one measure of non-uniqueness, the MSE between the original true hydraulic conductivities shown in the zoned Fig. 2 and those determined by the inversion, with the constraint that the inverse-solved values correspond to insignificant error in the head and age fields as measured by the sample locations described in Fig. 6. Note that no solution was found for the following cases due to non-convergence (1) 6 "true" observation of only head (2) 10 "noisy" observation of only head (3) 6 "noisy" observation of only head. The figure shows the reduction in this measure of non-uniqueness by the addition of the mean age data, as well as the reduction due to more data points sampled

absolute values of this number, and thus taller bars, means more unique inverse in terms of herein used measure of nonuniqueness. Figure 6 shows this measure of nonuniqueness:

- reduces due to the addition of the mean age data, even though the corresponding sample locations are near the upper boundary of the flow domain and not uniformly distributed.

- reduces due to addition of observation density when "true" observation values were used.

- reduces significantly due to addition of sampling points at the bottom of aquifer.

- increases due to the addition of data points when noisy observation values of both head and age observations were used, because they enter more error to the system and thus allow erroneous flow fields to produce perfect fit to the "noisy" observation values.

- The persistence of nonuniqueness is specifically due to the fact that the domain allows different flow fields to generate similar mean ages at sampling locations.

- When only head observations were used, the introduction of noise to the true observation values resulted in calibration procedure that does not converge.

As is clear from Fig. 6 and was discussed above, the addition of mean age observations reduced the level of nonuniqueness considerably, we hypothesize that this is a result of the fact that the age reflects the cumulative residence time of the water along streamlines and thus serves as a cumulative and not local measure of the flow properties. This renders age as a different and in some instance more powerful datum type than head which reflects local conditions through the diffusion operator. Nevertheless, the nonuniqueness persists because the heterogeneity of the domain allows different flow-fields to generate similar mean ages at the observation locations. This reflects the fact that the global nature of age does not guarantee uniqueness, as long as data are absent from locations where observations are needed to 
distinguish paths that give rise subsequently to similar ages downstream, but depart upstream. Thus, to address remaining nonuniqueness, age data at such observation locations are needed. The identification of these locations is an interesting research challenge.

These simple results reflect that distributed sampling location and combined used of different calibration targets (such as head and age) help to partially overcome the intrinsic nonuniqueness of the inverse problem.

\section{Summary}

There is a mechanistic basis for groundwater age as a deterministic distribution, and the basic Eq. 1 is the result. From this basic form some simplified results are obtained, including the fact that groundwater age is at least inverse-Gaussian distributed. The model can be generalized to formulate equations for multidomain transport problems and extant results for temporal moments as characteristic solutions to 1D transport all translate to results for age moments under same physical transport processes because the groundwater age equation reduces to the solute transport characteristic equation at steady state.

To summarize the inverse problem investigation, selection of appropriate observations density and observations type is important to reduce the level of calibration uncertainty. The addition of groundwater age observations in our study reduced the nonuniqueness of the calibration. The sampling density was also an important issue in our calibration, however the concentrated observation distribution was not able to overcome the nonuniqueness problem even in cases of dense sampling. Age observation was not able to eliminate nonuniqueness at locations far away from the sampling locations. Although application of distributed sampling enabled us to achieve unique estimated parameters using "true" observation values (subsampled directly from the forward solution), the same observation density/locations could not result in unique solution when random noises were added to the "true" observation values. Nonuniqueness specifically persists when the domain allows different flow fields to generate similar mean ages at sampling locations.

Acknowledgement This work supported by the UC Water Resources Center Research Program and by the DOE Environmental Remediation Science Program.

Open Access This article is distributed under the terms of the Creative Commons Attribution Noncommercial License which permits any noncommercial use, distribution, and reproduction in any medium, provided the original author(s) and source are credited.

\section{References}

Anderman, E.R., Hill, M.C., Poeter, E.P.: Two-dimensional advective transport in ground-water flow parameter estimation. Ground Water 34(6), 1001-1009 (1996). doi:10.1111/j.1745-6584.1996.tb02165.x

Bethke, C.M., Johnson, T.M.: Paradox of groundwater age. Geology 30(4), 385-388 (2002). correction doi:10.1130/0091-7613(2002)030<0386:POGAC >2.0.CO;2

Campaña, M.E.: Generation of ground-water age distributions. Ground Water 25, 51-58 (1987). doi:10.1111/j.1745-6584.1987.tb02115.x

Cheng, J.M., Yeh, W.W.G.: A proposed Quasi-Newton method for parameter-identification in a flow and transport-system. Adv. Water Resour. 15(4), 239-249 (1992). doi:10.1016/0309-1708(92)

Clarke, I.D., Fritz, P.: Environmental Isotopes in Hydrogeology, 352 pp. CRC, New York, NY (1997)

Cook, P.G., Böhlke, J.: Determining timescales for groundwater flow and solute transport. In: Cook, P. Herczeg, A. (eds), Environmental Tracers in Subsurface Hydrology, Kluwer, Boston (2000)

COMSOL Multiphysics Model Library, COMSOL Version 3.3, August, published by COMSOL AB, Palo Alto, CA, 362 pp (2006) 
Cornaton, F., Perrochet, P.: Groundwater age, life expectancy and transit time distributions in advective-dispersive systems: 1. Generalized reservoir theory. Adv. Water Resour. 29(9), 1267-1291 (2006). doi:10.1016/j.advwatres.2005.10.009

Cornaton, F., Perrochet, P.: Reply to "Comment on groundwater age, life expectancy and transit time distributions in advective-dispersive systems: 1 . Generalized reservoir theory" by Timothy R. Ginn. Adv. Water Resour. 30(4), 1058-1059 (2007). doi:10.1016/j.advwatres.2006.09.006

Danckwerts, P.V.: Continuous flow systems: Distribution of residence times. Chem. Eng. Sci. 2, 1-13 (1953). doi:10.1016/0009-2509(53)80001-1

Freeze, R.A., Witherspoon, P.A.: Theoretical analysis of regional groundwater flow. 2. Effect of water-table configuration and subsurface permeability variation. Water Resour. Res. 3(2), 623-634 (1967)

Ginn, T.R.: On the distribution of multicomponent mixtures over generalized exposure time in subsurface flow and reactive transport: Foundations, and formulations for groundwater age, chemical heterogeneity, and biodegradation. Water Resour. Res. 35(5), 1395-1407 (1999). doi:10.1029/1999WR900013

Ginn, T.R.: On the distribution of multicomponent mixtures over generalized exposure time in subsurface flow and reactive transport: Batch and column applications involving residence-time distributions and non-Markovian reaction kinetics. Water Resour. Res. 36(10), 2895-2903 (2000). doi:10.1029/ 2000WR900171

Ginn, T.R.: Stochastic-convective transport with nonlinear reactions and mixing: Finite streamtube ensemble formulation for multicomponent reaction systems with intra-streamtube dispersion. J. Contam. Hydrol. 47, 1-28 (2001). doi:10.1016/S0169-7722(00)00167-4

Ginn, T.R.: Comment on groundwater age, life expectancy and transit time distributions in advective-dispersive systems: 1. Generalized reservoir theory" by Timothy R. Ginn. Adv. Water Resour. 30(4), 1058-1059 (2007). doi:10.1016/j.advwatres.2006.09.005

Goode, D.J.: Direct simulation of groundwater age. Water Resour. Res. 32(2), 289-296 (1996). doi:10.1029/ 95WR03401

Haggerty, R.F., Gorelick, S.M.: Multiple-rate mass transfer for modeling diffusion and surface reactions in media with pore-scale heterogeneity. Water Resour. Res. 31(10), 2383-2400 (1995)

Harvey, C.F., Gorelick, S.M.: Mapping hydraulic conductivity—sequential conditioning with measurements of solute arrival time, hydraulic-head, and local conductivity. Water Resour. Res. 31(7), 1615-1626 (1995)

Hathhorn, W.E.: A second look at the method of random walks. Stoch. Hydrol. Hydraul. 10, 319-329 (1996). doi:10.1007/BF01581872

Hill, M.C., Tiedeman, C.R.: Effective groundwater model calibration: with analysis of data, sensitivities, predictions, and uncertainty, 455 pp. Wiley-Interscience, Hoboken, NJ (2007)

Ivey, S.S., Gentry, R.W., Larsen, D., Anderson, J.: Inverse application of age-distribution modeling using environmental tracers 3H/3He. J. Hydrol. Eng. 13(11), 1002-1010 (2008). doi:10.1061/ (ASCE)1084-0699(2008)13:11(1002)

Lassey, K.R.: Unidimensional solute transport incorporating equilibrium and rate-limited isotherms with firstorder loss 1. Model conceptualization and analytic solutions. Water Resour. Res. 24(3), 343-350 (1988). doi:10.1029/WR024i003p00343

Lassey, K.R.: Correction to "Unidimensional solute transport incorporating euqilibrium and rate-limited isotherms with first-order loss 1 . Model conceptualization and analytic solutions". Water Resour. Res. 25(3), 582 (1989). doi:10.1029/WR025i003p00582

Luo, J., Cirpka, O.A., Dentz, M., Carrera, J.: Temporal moments for transport with mass transfer described by an arbitrary memory function in heterogeneous media. Water Resour. Res. 44(1), W01502 (2008)

Maloszewski, P., Zuber, A.: Determining the turnover time of groundwater systems with the aid of environmental tracers 1: Models and their applicability. J. Hydrol. (Amst.) 57, 207-231 (1982). doi:10.1016/ 0022-1694(82)90147-0

Maloszewski, P., Zuber, A.: Manual on lumped parameter models used for the interpretation of environmental tracer dagta in groundwaters, in use of isotopes for analyses of flow and transport dynamics in groundwater systems, 1996-1999, IAEA, Vienna, Austria, 2002

Poeter, E.P., Hill, M.C., Banta, E.R., Mehl, S., Christensen, S.: UCODE_2005 and six other computer codes for universal sensitivity analysis, calibration, and uncertainty evaluation. U.S. Geological Survey, Techniques and Methods 6-A11, 283 pp (2005)

Portniaguine, O., Solomon, D.K.: Parameter estimation using groundwater age and head data, Cape Cod, MA. Water Resour. Res. 34(4), 637-645 (1998). doi:10.1029/97WR03361

Randolph, A.D.: A population balance for countable entities. Can. J. Chem. Eng. 42(December), 280-281 (1964)

Reilly, T.E., Plummer, L.N., Phillips, P.J., Busenburg, E.: The use of simulation and multiple environmental tracers to quantify groundwater-flow in a shallow aquifer. Water Resour. Res. 30(2), 421-433 (1994). doi:10.1029/93WR02655 
Reynolds, D.A., Marimuthu, S.: Deuterium composition and flow path analysis as additional calibration targets to calibrate groundwater flow simulation in a coastal wetlands system. Hydrogeol. J. 15(3), 515-535 (2007). doi:10.1007/s10040-006-0113-5

Scheibe, T.D., Chien, Y.J.: An evaluation of conditioning data for solute transport prediction. Ground Water 41(2), 128-141 (2003). doi:10.1111/j.1745-6584.2003.tb02577.x

Sheets, R.A., Bair, E.S., Rowe, G.L.: Use of H-3/He-3 ages to evaluate and improve groundwater flow models in a complex buried-valley aquifer. Water Resour. Res. 34(5), 1077-1089 (1998). doi:10.1029/98WR00007

Simmons, C.S.: A Stochastic-convective transport representation of dispersion in one-dimensional porous media systems. Water Resour. Res. 18(4), 1193-1214 (1982). doi:10.1029/WR018i004p01193

Spaulding, D.B.: A note on mean residence-times in steady flows of arbitrary complexity. Chem. Eng. Sci. 9, 74-77 (1958). doi:10.1016/0009-2509(58)87010-4

Sun, N.-Z.: Inverse Problems in Groundwater Modeling. Kluwer, Dordrecht (1994)

Sun, N.Z., Yeh, W.W.G.: Coupled inverse problems in groundwater modeling. 1. Sensitivity analysis and parameter-identification. Water Resour. Res. 26(10), 2507-2525 (1990)

Varni, M., Carrera, J.: Simulation of groundwater age distributions. Water Resour. Res. 34(12), 3271-3281 (1998). doi:10.1029/98WR02536

Weiss, R., Smith, L.: Parameter space methods in joint parameter estimation for groundwater flow models. Water Resour. Res. 34(4), 647-661 (1998). doi:10.1029/97WR03467

Weissmann, G.S., Zhang, Y., LaBolle, E.M., Fogg, G.E.: Dispersion of groundwater age in an alluvial aquifer system. Water Resour. Res. 38(10) (2002). doi:10.1029/2001WR000907

Wen, X.H., Deutsch, C.V., Cullick, A.S.: Construction of geostatistical aquifer models integrating dynamic flow and tracer data using inverse technique. J. Hydrol. (Amst.) 255(1-4), 151-168 (2002). doi:10.1016/ S0022-1694(01)00512-1

Woolfenden, L.R., Ginn, T.R.: Modeled ground water age distributions. Ground Water (2009) doi:10.1111/ j-1745-6584.2008.00550.x

Zhu, C.: Estimate of recharge from radiocarbon dating of groundwater and numerical flow and transport modeling. Water Resour. Res. 36(9), 2607-2620 (2000). doi:10.1029/2000WR900172 